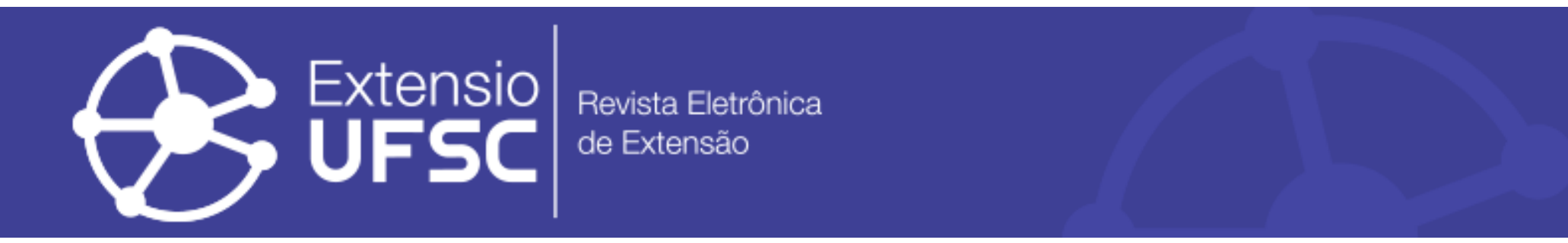

\title{
EDUCAÇÃO NUTRICIONAL SOBRE FÓRMULAS LÁCTEAS ARTIFICIAIS PARA GESTANTES DE RISCO: UM RELATO DE EXPERIÊNCIA
}

Tamires da Cunha Soares Universidade Federal do Piauí tamiressoares22@outlook.com

Diêla dos Santos Cunha Universidade Federal do Piauí dielasat@hotmail.com

Regina Márcia Soares Cavalcante Universidade Federal do Piauí reginalunna@hotmail.com

Resumo

Objetivo: Descrever a experiência da implementação de ações de educação nutricional sobre o uso de fórmulas artificiais para gestantes de risco atendidas em clínica pública especializada, da cidade de Picos-PI. Métodos: Após a realização de pesquisa diagnóstica, foram levantadas as principais dúvidas das gestantes, dentre as quais estava o uso de fórmulas infantis, respaldando a elaboração das ações de educação nutricional. A intervenção ocorreu durante encontros semanais que duravam aproximadamente 20 minutos, no período de um mês, na referida clínica. Foram utilizados cartazes ilustrativos. Resultados: As ações tiveram boa aceitação pelas gestantes, que participaram ativamente ao longo de seu desenvolvimento. Através da explanação dos conteúdos houve elucidação das principais dúvidas. Conclusão: Assim, anseia-se que a exposição deste relato de experiência sobre ações educativas em alimentação e nutrição, incentive a discussão sobre métodos de intervenção em saúde para que seus efeitos repercutam em melhorias na qualidade de vida da população.

Palavras-chave: Gravidez. Amamentação. Estudos de Intervenção. Fórmulas Infantis. Educação Alimentar e Nutricional.

\section{NUTRITION EDUCATION ABOUT ARTIFICIAL MILK FORMULAS FOR PREGNANT WOMEN AT RISK: AN EXPERIENCE REPORT}

\begin{abstract}
Objective: To describe the experience of the implementation of nutritional education actions about the use of artificial formulas for pregnant women at risk attended at a specialized public clinic in the city of Picos-PI. Methods: After the diagnostic research, the main doubts of the pregnant women were raised, among them was the use of infant formulas, supporting the elaboration of the nutritional education actions. The intervention occurred during weekly meetings that lasted approximately 20 minutes, in a period of one month, in the referred clinic. Illustrative posters were used. Results: The actions were well accepted by the pregnant women, who participated actively throughout their development. Through the explanation of the contents there was elucidation of the main doubts. Conclusion: Thus, it is hoped that the exposure of this experience report on educational actions in food and nutrition, encourage the discussion on methods of health intervention so that their effects will have repercussions on the quality of life of the population.
\end{abstract}

Keywords: Pregnancy. Breast-feeding. Clinical Trial. lnfant Formula. Food and Nutrition Education.

\section{EDUCACIÓN NUTRICIONAL SOBRE FÓRMULAS LÁCTEAS ARTIFICIALES PARA MUJERES EMBARAZADAS DE RIESGO: UM REALATO DE EXPERIENCIA}

\section{Resumen}

Objetivo: Describir la experiencia de la implementación de acciones de educación nutricional sobre el uso de fórmulas artificiales para mujeres embarazadas de riesgo atendidas en clínica pública especializada, de la ciudad de Picos-PI. Métodos: Después de la realización de investigación diagnóstica, fueran investigadas las principales dudas de las mujeres, entre las cuales estaba el uso de fórmulas infantiles, que fueron la base para elaboración de las acciones de educación nutricional. La intervención ocurrió durante encuentros semanales que duraban aproximadamente 20 minutos, en el período de um mes, en la referida clínica. Se utilizaron carteles con ilustraciones. Resultados: Las acciones tuvieron buena aceptación por las gestantes, que participaron activamente a lo largo de su desarrollo. Por medio de la explicación de los contenidos hubo elucidación de las principales dudas. Conclusión: Así, se espera que la exposición de este relato de experiencia sobre acciones educativas en alimentación y nutrición, incentive la discusión sobre métodos de intervención en salud para que sus efectos repercutan en mejoras en la calidad de vida de la población.

Palabras clave: Embarazo. La lactancia Materna. Estudios de Intervención. Fórmulas Infantiles. Educación Alimentaria y Nutricional. 
Educação nutricional sobre fórmulas lácteas artificiais para gestantes de risco: um relato de experiência

\section{INTRODUÇÃO}

A Educação Nutricional busca promover a segurança alimentar e nutricional através de ações educativas sobre práticas alimentares adequadas que permitem às pessoas a seleção e consumo de alimentos saudáveis e nutritivos, além da valorização da diversidade de produtos regionais (KURZ; MORGAN; TENROLLER, 2012).

A gestação é um fenômeno natural que envolve uma série de modificações metabólicas, fisiológicas e eventos que intervêm na vida da mulher, como os períodos de lactação e puerpério. De acordo com o ponto de vista da biomedicina, é evidente que são fases de maior vulnerabilidade e de grandes demandas que solicitam prioridade na assistência. Portanto, ações educativas em saúde desenvolvidas durante e após o período gestacional podem influenciar a saúde da gestante e do feto, diminuindo riscos de mortalidade infantil (BAIÃO; DESLANDES, 2006; VITOLO, 2008).

A amamentação compreende uma das etapas mais significativas no processo reprodutivo da mulher, sua execução oferece benefícios tanto para mãe como para o recém-nascido. $O$ aleitamento materno exclusivo, provê alimento ao filho e ainda promove a saúde da mãe, devendo ser ofertado durante os seis primeiros meses de forma exclusiva, e posteriormente, acompanhado da introdução de alimentos complementares apropriados, garantindo assim, inúmeros benefícios nutricionais, imunológicos e afetivos, considerado de fundamental importância para a saúde, bem como, sobrevivência das crianças (VITOLO, 2008; MARTINS; SANTANA, 2013; RAMOS et al., 2010).

No entanto, algumas situações podem impossibilitar o aleitamento materno e o lactente necessitará receber a fórmula infantil que poderá suprir as necessidades nutricionais e manter o bom estado fisiológico da criança em seu primeiro ano de vida. Fórmulas lácteas para lactentes são definidas pela Agência Nacional de Vigilância Sanitária como produtos apresentados em forma de pó ou líquido, que devem ser utilizados sob prescrição, especialmente desenvolvido para suprir, por si só, as demandas nutricionais de crianças sadias durante os primeiros seis meses de vida (KUS et al., 2011; MEIRELLES et al., 2008; BRASIL, 2011).

Neste caso, ações de educação nutricional terão como objetivo levar conhecimento sobre as indicações pertinentes da utilização de fórmulas lácteas, para se evitar o consumo desnecessário destes produtos, que embora supram de forma satisfatória as necessidades nutricionais, não se equiparam a qualidade do leite materno. Além disso, se busca fornecer instrução sobre a correta manipulação e higienização para os procedimentos de preparo. 
Educação nutricional sobre fórmulas lácteas artificiais para gestantes de risco: um relato de experiência

Diante da importância da educação nutricional para garantir o alcance das necessidades nutricionais do recém-nascido, tendo em vista que as futuras mães devem conhecer a respeito da correta indicação de uso de fórmulas lácteas artificiais, justificou-se a realização desse estudo no sentido de relatar a experiência sobre a implementação de ações educativas que visaram esclarecer as principais dúvidas apresentadas pelas gestantes quanto as aspectos relativos ao uso de fórmulas infantis.

\section{MATERIAIS E MÉTODOS}

Trata-se de uma descrição de experiência de ações de Educação Nutricional, desenvolvida em uma Clínica Integrada de Saúde da Mulher (CLISAM), localizada na área urbana do município de Picos, estado do Piauí. As ações de educação nutricional integram um projeto de extensão, intitulado "Educação Nutricional Continuada para Gestantes de Risco", o projeto é constituído por acadêmicas do $8^{\circ}$ e $9^{\circ}$ períodos do curso de nutrição da Universidade Federal do Piauí, sob orientação da docente responsável.

Para a realização do estudo, que ocorreu de março a outubro de 2016, foi desenvolvido e aplicado um questionário estruturado, pelas próprias pesquisadoras na referida clínica, para coleta de informações sociodemograficas como idade, escolaridade, renda e ainda informações sobre as principais dúvidas durante e após a gestação.

Posteriormente realizou-se uma intervenção na clínica abordando o tema "Uso de Leites Artificiais", havendo uma breve discussão sobre quando e por que utiliza-los, malefícios para os lactentes e lactantes e cuidados com a higiene antes, durante e depois do preparo. A intervenção foi realizada por meio de miniexposições dialogadas no período de um mês, sendo realizadas uma vez por semana com auxílio de cartazes, durante aproximadamente 20 minutos, antes do atendimento médico. Os cartazes foram confeccionados pelas participantes do projeto sob orientação da coordenadora do mesmo.

\section{RESULTADOS E ANÁLISES}


Educação nutricional sobre fórmulas lácteas artificiais para gestantes de risco: um relato de experiência

Após realização da avaliação diagnóstica, pôde-se delimitar o perfil da população alvo do estudo observando-se, também, as dúvidas mais comuns relatadas entre as gestantes de risco. Foram entrevistadas 18 gestantes, com idade média de 25,7 anos, sendo que a idade mínima apresentada foi de 16 anos e a máxima de 35 anos. Todas tinham baixas escolaridade e renda.

Quanto as dúvidas relacionadas a alimentação e nutrição, foram relatadas com mais frequência: alimentação complementar; uso de leites artificiais; uso de mamadeiras e chupetas; suplementação de ácido fólico; e alimentação adequada na gestação. As demais temáticas também resultaram em abordagens educativas, porém, este trabalho abordará especificamente o uso de leites artificiais.

A partir das dúvidas das gestantes foram elaboradas ações de educação alimentar e confeccionados cartazes, para realização de intervenções nutricionais com os seguintes temas: Leite artificial: quando utilizar?; Condições maternas que podem justificar a não amamentação; Leite artificial: benefícios e malefícios para a mamãe e para o bebê; Limpeza e higiene; e Recomendações para evitar contaminação de leites artificiais. As ações foram realizadas em dias diferentes e com gestantes distintas, todos os temas foram abordados, de forma simples e dinâmica, a cada encontro com a finalidade de que o maior número possível de gestantes tivessem acesso ao conteúdo.

Para a confecção do material de exposição, prezou-se pela ilustração com figuras que representassem bem o tema e frases curtas e de fácil compreensão, para facilitar a assimilação e chamar a atenção das gestantes para os assuntos abordados. As exposições ocorreram em forma de roda de conversa, com a participação ativa das mulheres presentes. Ao final de cada encontro, foram realizadas dinâmicas de grupo com perguntas e respostas, para verificar a fixação dos conteúdos e solucionar as dúvidas remanescentes.

\section{Primeiro tema: Condições neonatais que justificam o uso de fórmulas lácteas}

No assunto inicial, foi apresentado às gestantes no cartaz que abordava o tema "Leite artificial: quando utilizar?" mostrando situações neonatais que justificavam a utilização de fórmulas lácteas artificiais no lugar do leite materno.

De acordo com Melo e Gonçalves (2014) em circunstâncias em que o aleitamento materno não é possível, é recomendado o uso de fórmulas lácteas modificadas para lactentes, que embora difira do leite materno quanto a propriedades imunológicas e digestivas, atendem as necessidades nutricionais estimadas.

O cartaz utilizado neste encontro, abordou os subtemas galactosemia, fenilcetonúria, intolerância à lactose, lactentes com risco de hipoglicemia e portadores de síndrome onde haja 
Educação nutricional sobre fórmulas lácteas artificiais para gestantes de risco: um relato de experiência

dificuldade de sucção, nascidos com menos de $1500 \mathrm{~g}$ e nascidos com idade gestacional inferior a 32 semana, ou que não conseguem ganhar peso ou manter o grau de hidratação só com o leite materno, o uso de fórmulas ocorrerá por tempo limitado. Segundo Volo et al. (2013), estes casos específicos justificam a utilização de fórmulas infantis. É importante acrescentar que visou-se sempre explanar os termos técnicos em uma forma de linguagem mais acessível àquela população, exemplificando e trazendo para a realidade da mesma, o que possibilitou o bom entendimento das futuras mães sobre o tema.

\section{Segundo tema: Condições maternas que justificam o uso de fórmulas lácteas}

Em seguida, foi apresentado o tema "Condições maternas que podem justificar a não amamentação". O cartaz utilizado trouxe infecções por HIV, baixa produção láctea, quimioterapia, sepsis, consumo de drogas lícitas e/ou ilícitas e uso de medicamentos incompatíveis com a amamentação. O tema foi retratado de forma bem sucedida, através de imagens ilustrativas com breves frases referentes as condições em que são justificadas a não amamentação, com cuidado ao tratar de temas que pudessem ser considerados delicados para algumas mães.

Segundo Meirelles e colaboradores (2008), os subtemas abordados são condições médicas relativas à mãe, listadas na recomendação internacional de utilização de fórmulas lácteas. Ainda de acordo com Machado e colaboradores (2007), o aleitamento materno constitui-se como uma possível causa de transmissão do vírus de imunodeficiência adquirida (HIV), nestes casos, o uso de fórmulas lácteas infantis seria, igualmente, justificado.

Além disso, algumas intercorrências comuns no início da amamentação como traumas mamilares podem dificultar este processo, como ingurgitamento mamário e mastite, onde mesmo que haja a produção do leite materno, se faz necessário o consumo de leites artificiais, mesmo que temporariamente (PARADA et al., 2005).

\section{Terceiro tema: Malefícios do uso de fórmulas lácteas}

O terceiro cartaz ilustrou o tema "Leite artificial: malefícios para a mamãe e para o bebê", que tratou das possíveis consequências negativas que o consumo de leites artificiais pode trazer, quando sua utilização não é realmente necessária. As situações abordadas englobaram prejuízos afetivos, possibilidade aumentada de diarreias, infecções respiratórias e contaminações do leite durante o preparo.

O estudo de Melo e Gonçalves (2014), ressaltou a importância da amamentação para estabelecer o vínculo afetivo entre mãe e filho, a utilização de fórmula poderá interferir neste 
Educação nutricional sobre fórmulas lácteas artificiais para gestantes de risco: um relato de experiência

desenvolvimento. Além disso, o uso de fórmulas pode trazer episódios frequentes de diarreias e até mesmo infecções respiratórias, aumentando a frequência de desnutrição e diabetes, e maior risco de contaminação dos leites artificiais. O estudo observou também, que é relatado um menor espaço entre gravidezes, maiores incidências de tumores e depressão maternas e estes achados também foram abordados no encontro.

No momento da dinâmica de fixação deste encontro em especial, algumas gestantes se mostraram surpresas com a possibilidade de prejuízos ao se utilizar estes produtos. O principal desafio desta abordagem foi não passar uma visão de insegurança para as mães que porventura viessem precisar utilizar estes produtos, mas reforçar que este consumo deve ocorrer apenas quando necessário e não de forma discriminada e aleatória.

\section{Quarto tema: Importância da higiene na preparação de fórmulas lácteas}

Posteriormente, tratou-se a temática "Limpeza e higiene", que foi abordado trazendo medidas sanitárias a serem adotadas antes e durante a preparação das fórmulas artificias. Os cuidados citados foram lavagem das mãos antes do preparo de mamadeiras, ferver a água utilizada no preparo das mamadeiras, mesmo que esta seja filtrada e esterilizar as mamadeiras e bicos.

A Organização Mundial de Saúde (2015), em manual que aborda boas práticas de preparação, manipulação e conservação de fórmulas desidratadas para lactentes, com as tecnologias atualmente existentes, a indústria ainda não pôde produzir leites artificias estéreis para o consumo, a incorreta manipulação durante o preparo pode agravar ainda mais o problema. As recomendações abordadas neste encontro fazem parte das medidas aconselhadas pela OMS para se minimizar estes riscos.

Durante a dinâmica de fixação, além das perguntas realizadas, foi realizada a demonstração da correta lavagem das mãos para se evitar contaminações durantes o preparo das fórmulas. Ao serem indagadas oralmente, as gestantes consideraram este um tema muito importante e de fácil assimilação.

\section{Quinto tema: Recomendações gerais de preparo}

O último cartaz trouxe "Recomendações para evitar contaminação de leites artificiais", onde foram abordadas algumas orientações básicas a se seguir a fim de evitar contaminação da fórmula já pronta para o consumo. O cartaz trouxe os tópicos baseados nas recomendações da Organização Mundial da Saúde (2015), que são: não utilizar jarra térmica para conservar o leite quente, não guardar o restante do leite não consumido para depois, não reaquecer o mesmo leite, 
Educação nutricional sobre fórmulas lácteas artificiais para gestantes de risco: um relato de experiência

não deixa-lo em temperatura ambiente por mais que alguns minutos e recomendações para viagem, como levar água morna em água térmica para preparo imediato quando precisar.

O preparo do leite artificial deve ocorrer para seu consumo imediato, logo, sob nenhuma hipótese se deve armazená-lo ou utilizar um leite preparado há um período de tempo superior a alguns minutos, pois a contaminação deste alimento poderia resultar em quadros infecciosos que foram listados na temática "Malefícios do uso de fórmulas lácteas” (WHO, 2015).

A interação das gestantes com o tema foi positiva, muitas delas classificaram alguns pontos como novos e que a adoção dessas práticas poderá garantir mais segurança ao seu filho, caso este venha precisar utilizar leites artificiais.

\section{Momento pós-explanação}

Após a explanação dos temas, foram realizadas dinâmicas com perguntas rápidas e orais para verificar se houve, de fato, a assimilação dos conteúdos abordados e pode-se perceber uma melhor compreensão por parte delas. Ademais, as gestantes tiveram liberdade para relatar suas vivências e fazer indagações, caso algum ponto ainda não estivesse satisfatoriamente claro para elas. Foi aberto espaço para colocações e debates sobre o tema, a fim de valorizar os conhecimentos que as gestantes já detinham sobre os assuntos discutidos e promover um ambiente favorável de troca de experiências.

\section{CONSIDERAÇÕES FINAIS}

A realização das ações educativas tiveram potencial para produzir efeitos positivos junto às gestantes de risco, pois houve o esclarecimento de dúvidas apresentadas pelas próprias gestantes sobre o correto uso de fórmulas lácteas artificiais. 
Educação nutricional sobre fórmulas lácteas artificiais para gestantes de risco: um relato de experiência

O conhecimento sobre condições materno-infantis que impossibilitam a amamentação e malefícios do uso indevido de fórmulas infantis, poderão evitar o uso desnecessário das mesmas. Ademais, a adoção de boas práticas de manipulação destes leites, diminuirá a probabilidade de contaminações, garantindo uma maior segurança ao lactente. Entretanto, estudos mais conclusivos, com utilização de questionário estruturados sobre a fixação dos conteúdos abordados, devem ser realizados para avaliar o grau de efetividade da intervenção aqui descrita.

Pode-se ressaltar que a educação nutricional é utilizada como mecanismo de empoderamento da população, que uma vez conhecendo os procedimentos corretos de utilização de fórmulas lácteas, terá a possibilidade de fazer boas escolhas à luz de recomendações profissionais.

Enfim, o estímulo ao diálogo e reflexão sobre temas de saúde, gera a sensibilização da população em geral, propiciando mudanças de hábitos de vida que trazem melhorias reais aos indivíduos. Anseia-se que a exposição do relato de experiência sobre ações educativas em alimentação e nutrição estimulem a discussão sobre métodos de intervenção em saúde, a fim de que seus efeitos sejam sentidos em forma de melhorias na qualidade de vida.

\section{REFERÊNCIAS}

BAIÃO, M. R.; DESLANDES, S. F. Alimentação na gestação e puerpério. Revista de Nutrição, v. 19, n. 2, p. 245-253, 2006.

BRASIL. Ministério da Saúde. Agência Nacional de Vigilância Sanitária. Resolução RDC n ${ }^{\circ} 43$, de 19 de setembro de 2011. Dispõe sobre o regulamento técnico para fórmulas infantis para lactentes. Disponível em: <https://www.saude.rj.gov.br/comum/code/MostrarArquivo.php?C=MTk4MQ\%2C\%2C $>$. Acesso em 14/06/2018.

KUS, M. M.; SILVA, S. A.; AUED-PIMENTEL, S.; MANCINI-FILHO, J. Informação nutricional de fórmulas infantis comercializadas no estado de São Paulo. Revista de Nutrição, v. 24, n. 2, p. 209-218, 2011.

MACHADO, M. M. T.; GALVÃO, M. T. G.; KERR-PONTES, L. R. S.; CUNHA, A. J. L. A.; LEITE, A. J. M.; LINDSAY, A. C.; LEITE, R. D.; LEITE, C. A. C. Acesso e utilização de fórmula infantil e alimentos entre crianças nascidas de mulheres com HIV/AIDS. Revista Eletrônica de Enfermagem, v. 9, n. 3, p. 699-711, 2007.

MARTINS, M. Z.; SANTANA, L. S. Benefícios da amamentação para saúde materna. Interfaces Cientificas, v. 1, n. 3, p. 87-97, 2013.

MEIRELLES, C. A. B.; OLIVEIRA, M. I. C.; MELLO, R. R.; VARELA, M. A. B.; FONSECA, V. M. Justificativas para o uso de suplemento em recém-nascidos de baixo risco de um Hospital Amigo da Criança. Cadernos de Saúde Pública, v. 24, n. 9, p. 2001-2012, 2008. 
Educação nutricional sobre fórmulas lácteas artificiais para gestantes de risco: um relato de experiência

MELO, C. S.; GONÇALVES, R. M. Aleitamento materno versus aleitamento artificial. Estudos, v. 41, n. 1, p. 7-14, 2014.

RAMOS, C. V.; ALMEIDA, J. A. G.; SALDIVA, S. R. D. M.; PEREIRA, L. M. R.; ALBERTO, N. S. M. C.; TELES, J. B. M.; PEREIRA, T.G. Prevalência do aleitamento materno exclusivo e os fatores a ele associados em crianças nascidas nos Hospitais Amigos da Criança de Teresina Piauí. Epidemiologia e Serviços de Saúde, v. 19, n. 2, p. 115-124, 2010.

SANTOS, L. A.; MAMEDE, F. V.; CLAPIS, M. J.; BERNARDI, J. V. B. Orientação nutricional no pré-natal em serviços públicos de saúde no município de Ribeirão Preto: o discurso e a prática assistencial. Revista Latino-Americana de Enfermagem, v. 14, n. 5, p. 1-7, 2006.

VITOLO. M. R. Da gestação ao envelhecimento. Rio de Janeiro: Editora Rúbio. 2008. 648 p.

VOLO, A. S. C.; ARAÚJO, E. B.; CARVALHO, M. I. S.; MAIA, W. O.; SOUZA, E. B.; GROSSMANN, S. M. C. Avaliação da adequada indicação de leite artificial em recém nascidos em uma maternidade de referência de Minas Gerais. Revista Universidade Vale do Rio Verde, v. 11, n. 1, p.78-83, 2013.

World Health Organization. Preparação, manipulação e conservação de fórmulas desidratadas para lactentes: Manual de boas práticas. Portugal: Instituto Nacional de Saúde Doutor Ricardo Jorge, 2015. 26p.

Recebido em: 07/10/2017

Aceito em: 28/06/2018 